\title{
Experimental and theoretical studies of the reactions of ground-state sulfur atoms with hydrogen and deuterium
}

\author{
Kristopher M. Thompson, ${ }^{1}$ Yide Gao, ${ }^{1, a}$ ) Paul Marshall, ${ }^{1, b)}$ Han Wang, ${ }^{2}$ Linsen Zhou, ${ }^{3}$ \\ Yongle $\mathrm{Li}^{2}{ }^{2, \mathrm{C})}$ and $\mathrm{Hua} \mathrm{Guo}^{3}$ \\ ${ }^{1}$ Department of Chemistry, University of North Texas, Denton, Texas 76203, USA \\ ${ }^{2}$ Department of Physics, International Center of Quantum and Molecular Structures, and Shanghai Key \\ Laboratory of High Temperature Superconductors, Shanghai University, Shanghai 200444, China \\ ${ }^{3}$ Department of Chemistry and Chemical Biology, University of New Mexico, Albuquerque, \\ New Mexico 87131, USA
}

(Received 20 June 2017; accepted 12 September 2017; published online 3 October 2017)

\begin{abstract}
The gas-phase kinetics of $\mathrm{S}\left({ }^{3} \mathrm{P}\right)$ atoms with $\mathrm{H}_{2}$ and $\mathrm{D}_{2}$ have been studied via the laser flash photolysis-resonance fluorescence technique. $\mathrm{S}$ atoms were generated by pulsed photolysis of $\mathrm{CS}_{2}$ at $193 \mathrm{~nm}$ and monitored by time-resolved fluorescence at $181 \mathrm{~nm}$. The rate coefficients for $\mathrm{H}_{2}\left(k_{1}\right)$ and $\mathrm{D}_{2}\left(k_{2}\right)$, respectively, are summarized as $k_{1}(600-1110 \mathrm{~K})=3.0 \times 10^{-9}$ $\exp \left(-\frac{1.317 \times 10^{5}-2.703 \times 10^{7} \mathrm{~K} / T}{8.314 T / \mathrm{K}}\right) \mathrm{cm}^{3}$ molecule $^{-1} \mathrm{~s}^{-1}$ and $k_{2}(770-1110 \mathrm{~K})=2.2 \times 10^{-14}(T / 298 \mathrm{~K})^{3.55}$ $\exp (-5420 \mathrm{~K} / T) \mathrm{cm}^{3}$ molecule $\mathrm{s}^{-1}$. Error limits are discussed in the text. The rate coefficients for formation of $\mathrm{SH}(\mathrm{SD})+\mathrm{H}(\mathrm{D})$ on a newly developed triplet potential energy surface were characterized via ring polymer molecular dynamics and canonical variational transition-state theory. There is excellent agreement above about $1000 \mathrm{~K}$ between theory and experiment. At lower temperatures, the experimental rate coefficient is substantially larger than the results computed for the adiabatic reaction, suggesting a significant role for intersystem crossing to the singlet potential energy surface at lower temperatures. Published by AIP Publishing. https://doi.org/10.1063/1.4991418
\end{abstract}

\section{INTRODUCTION}

Fossil fuels typically contain up to several per cent of sulfur, and during combustion, sulfur chemistry can influence the radical pool and impact the extent to which nitrogen oxides are formed. ${ }^{1,2}$ Much of the high-temperature chemistry involved remains unknown. Sulfur-hydrogen interactions are of particular significance for modeling the Claus process to remove sulfur from hydrocarbon fuels. ${ }^{3}$ Here, we report measurements of the rate coefficient $k_{1}$ for the reaction

$$
\mathrm{S}\left({ }^{3} \mathrm{P}\right)+\mathrm{H}_{2} \rightarrow \text { products }
$$

which broaden the range of temperature previously accessed by shock tube experiments. ${ }^{4,5}$ Reaction (1) is also of fundamental interest as a small system that may exhibit intersystem crossing (ISC). Shiina et al. have noted that a reaction may occur on the triplet potential energy surface (PES) via a barrier to yield $\mathrm{H}+\mathrm{SH}$ or that ISC may occur leading to singlet $\mathrm{H}_{2} \mathrm{~S} .{ }^{6}$ Maiti et al. have explored the coupling between triplet and singlet PESs in detail but did not report rate coefficients. ${ }^{7}$ Such coupling may also be important in the unimolecular decomposition of $\mathrm{H}_{2} \mathrm{~S}$, which was initially thought to proceed analogously to the decomposition of water to $\mathrm{H}+\mathrm{SH}^{4,8-10}$ but now appears to lead in art $^{3}$ or fully ${ }^{11,12}$ to $\mathrm{S}+\mathrm{H}_{2}$. The impact

\footnotetext{
a)Present address: PPG Industries, 3333 N Interstate 35, Gainesville, Texas 76240, USA.

b)Electronic mail: marshall@unt.edu

c)Electronic mail: yongleli@shu.edu.cn
}

of ISC on kinetics is likely to be more pronounced at low temperatures due to the facts that the singlet-triplet crossing seam is energetically much lower than the triplet barrier and that the singlet PES is barrierless. ${ }^{7}$ As a result, the ISC effect is hard to delineate from the influence of tunneling. Here we employ a newly developed triplet PES using the high-level $a b$ initio data reported in a recent publication ${ }^{13}$ to investigate the kinetics of reaction (1) using ring polymer molecular dynamics (RPMD) rate theory. ${ }^{14}$ The RPMD approach enables the inclusion of quantum mechanical zero-point energy and tunneling which are expected to be significant for these heavy-light-light systems involving hydrogens. As a further test of theory, we have determined $k_{2}$ for

$$
\mathrm{S}\left({ }^{3} \mathrm{P}\right)+\mathrm{D}_{2} \rightarrow \text { products }
$$

and extended existing measurements ${ }^{15}$ to lower temperatures where tunneling or ISC may become important. The comparison between the experimental data and the singlesurface theoretical rate coefficients with the inclusion of tunneling will thus shed light on the importance of ISC in these reactions.

\section{METHODOLOGY}

\section{A. Experiments}

Mixtures of $\mathrm{CS}_{2}$ (Sigma-Aldrich, 99\%, trapped and degassed at $196 \mathrm{~K})$ in $\mathrm{Ar}\left(99.9999 \%\right.$, Air Liquide) and $\mathrm{H}_{2}$ (MG Industries, ultra high purity $99.999 \%,<1 \mathrm{ppm} \mathrm{O}_{2}$ ) or $\mathrm{D}_{2}$ (Matheson, research purity, chemical purity $99.999 \%$, isotopic 
purity $99.8 \%,<1 \mathrm{ppm}_{2}$ ) in Ar were prepared manometrically in glass bulbs. Flows of these mixtures were measured and combined before they entered the stainless steel reaction cell. The total pressure, dominated by Ar, was measured with a capacitance manometer and the temperature in the reaction zone, where the photolysis and probe beams intersect, with a retractable thermocouple corrected for radiation errors. ${ }^{16}$ Ground-state sulfur atoms were generated by pulsed photolysis of the $\mathrm{CS}_{2}$ precursor and then monitored as they reacted with an excess of $\mathrm{H}_{2}$ or $\mathrm{D}_{2}$. Details of the apparatus and its operation have been provided elsewhere. ${ }^{17,18} \mathrm{In}$ brief, excimer laser pulses at $193 \mathrm{~nm}$ dissociated some of the $\mathrm{CS}_{2}$. The Ar bath gas served to quench any excited singlet $\mathrm{S}$ atoms formed within $1 \mu \mathrm{s},{ }^{19}$ to maintain thermal equilibrium during the reaction, and to slow diffusion of radicals to the surfaces of the reaction cell, which is therefore effectively wall-less. Atomic sulfur was monitored by resonance fluorescence in the vacuum ultraviolet region, $(4 \mathrm{~s}){ }^{3} \mathrm{~S} \rightarrow(3 \mathrm{p}){ }^{3} \mathrm{P}$ at $\lambda \approx 181 \mathrm{~nm}$, excited by a microwave-discharge through ca. $0.01 \% \mathrm{H}_{2} \mathrm{~S}$ in $\mathrm{Ar}$ and detected with a solar-blind photomultiplier tube operated with photon counting. The laser pulses were repeated at ca. 1-2 Hz, and time-resolved signals following up to 1000 pulses were accumulated in a multichannel scaler. These fluorescence signals are proportional to [S], plus a constant that reflects a steady background of scattered light.

The rate law

$\frac{d[\mathrm{~S}]}{d t}=-k_{1}[\mathrm{~S}]\left[\mathrm{H}_{2}\right]-k_{\text {diff }}[\mathrm{S}]-k_{\mathrm{CS}_{2}}[\mathrm{~S}]\left[\mathrm{CS}_{2}\right]=-k_{p s 1}[\mathrm{~S}]$

leads to exponential decays. Here, $k_{\text {diff }}$ accounts for effectively first-order diffusional loss of $\mathrm{S}$ and $k_{\mathrm{CS}_{2}}$ describes the reaction of $\mathrm{S}$ atoms with the precursor, ${ }^{20}$ whose concentration is held constant as $\left[\mathrm{H}_{2}\right]$ is varied. $k_{p s 1}$ is the pseudo-first-order decay coefficient when $\left[\mathrm{H}_{2}\right] \gg[\mathrm{S}]$. Inspection of the residuals while fitting the observed decays verified that any deviations were within the scatter expected from Poisson statistics of the photon counts. At each set of conditions, five decays were characterized with $[\mathrm{S}]$ from 0 to $\left[\mathrm{H}_{2}\right]_{\max }$, and $k_{1}$ was derived from the slope of a plot of $k_{p s} 1$ vs. [ $\left.\mathrm{H}_{2}\right]$. This procedure was repeated at various temperatures to determine $k_{1}(T)$ and at various total pressures $P$ to determine if there was any dependence of $k_{1}$ on [Ar]. Variation of the average residence time within the heated reactor before photolysis, $\tau_{\text {res }}$, checks for any decomposition of the gas mixtures. The UV laser fluence $F$ was obtained from the pulse energy, the beam cross section of $0.6 \mathrm{~cm}^{2}$, and the window transmittance measured to be 0.83 . Changes in $\left[\mathrm{CS}_{2}\right]$ and $F$ alter the initial concentration of radicals generated photolytically. $F$ can also be combined with the absorption cross section of $\mathrm{CS}_{2}$ to estimate the initial absolute $[\mathrm{S}],{ }^{19}$ although relative values are sufficient for application of Eq. (3).

\section{B. Ring polymer molecular dynamics}

An approximate quantum mechanical simulation method, ring polymer molecular dynamics (RPMD), is used to compute rate coefficients of the title reactions, assuming that the reactions proceed adiabatically on the $1^{3} A^{\prime \prime}$ state PES. Introduced in a heuristic fashion, RPMD takes advantage of the isomorphism between the quantum statistical mechanics of a physical system and its classical counterpart, by mapping each atom to a ring polymer consisting of fictitious classical beads connected by harmonic potentials. ${ }^{21}$ Such a mapping is numerically advantageous because the propagation of classical trajectories is relatively straightforward and has favorable scaling laws. The RPMD framework has been adapted for calculating rate coefficients, using the correlation function approach. ${ }^{22-25}$ There are several distinct advantages with the RPMD rate theory. ${ }^{14,26}$ For instance, RPMD is reduced to classical molecular dynamics when only one bead is included. As a result, it converges to classical transition-state theory (TST) results at high temperatures. ${ }^{27}$ On the other hand, it can approximately capture quantum effects in the reaction rate coefficients, such as zero-point energy (ZPE) and tunneling ${ }^{22}$ so that the rate coefficients obtained by RPMD are also quite accurate at low temperatures where conventional TST may not provide reliable results. Finally, there is no need to optimize the dividing surface since the influence of the dividing surface location is counterbalanced by the transmission coefficient. ${ }^{23}$ That is a highly desirable property due to the difficulty of defining the dividing surface in highdimensional systems. More recently, it has been shown that the RPMD rate theory is related to quantum transition-state theory. ${ }^{28,29}$ The RPMD rate theory has been extensively validated against accurate quantum dynamics results in a number of systems. $^{14}$

The Hamiltonian of the title reaction can be written in atomic Cartesian coordinates as follows:

$$
\hat{H}=\sum_{i=1}^{3} \frac{\left|\hat{p}_{i}\right|^{2}}{2 m_{i}}+V\left(\hat{q}_{1}, \hat{q}_{2}, \hat{q}_{3}\right),
$$

where $\hat{p}_{i}, \hat{q}_{i}$, and $m_{i}$ stand for the momentum operator, position operator, and mass of the $i_{\text {th }}$ atom, respectively. By replacing each atom with a closed ring polymer with $n$ beads, the RPMD Hamiltonian assumes the form below

$$
\begin{aligned}
H_{n}(\vec{p}, \vec{q})= & \sum_{i=1}^{3} \sum_{j=1}^{n}\left(\left|\frac{\left.\vec{p}_{i}^{(j)}\right|^{2}}{2 m_{i}}+\frac{m_{i} \omega_{n}^{2}}{2}\right| \vec{q}_{i}^{(j)}-\left.\vec{q}_{i}^{(j-1)}\right|^{2}\right) \\
& +\sum_{j=1}^{n} V\left(\vec{q}_{1}^{(j)}, \vec{q}_{2}^{(j)}, \vec{q}_{3}^{(j)}\right)
\end{aligned}
$$

where $\vec{q}_{i}^{(0)}=\vec{q}_{i}^{(n)}$ for the $i_{\text {th }}$ atom. The force constant between adjacent beads is $\omega_{n}=\left(\beta_{n} \hbar\right)^{-1}$ with the reciprocal temperature of the system $\beta_{n}=\left(n k_{B} T\right)^{-1}$ ( $k_{B}$ is the Boltzmann constant).

To describe the extent of reaction, a pair of dividing surfaces is defined in terms of the centroids of the ring polymer $(\overline{\vec{q}})$. The first dividing surface is placed in the reactant asymptote, ${ }^{26}$

$$
s_{0}(\overline{\bar{q}})=R_{\infty}-|\bar{R}|,
$$

where $|\bar{R}|$ is the centroid length of the vector that connects the centers of mass of the two reactants and $R_{\infty}$ is chosen to be large enough to make sure that the interaction between the reactants is negligible. The second dividing surface is placed in the transition-state region on the PES, which is at a collinear 
$\mathrm{S}-\mathrm{H}-\mathrm{H}$ configuration (vide infra). There are two equivalent product arrangement channels, and the dividing surface is defined as follows: ${ }^{26}$

$$
s_{1}(\overline{\vec{q}})=\max \left\{s_{1 \alpha}(\overline{\bar{q}}), s_{1 \beta}(\overline{\bar{q}})\right\},
$$

where

$$
s_{1 x}(\overline{\bar{q}})=\left(\left|\overrightarrow{\vec{q}}_{H H_{x}}\right|-q_{H H_{x}}^{\neq}\right)-\left(\left|\overline{\vec{q}}_{S H_{x}}\right|-q_{S H_{x}}^{\neq}\right), x=\alpha, \beta .
$$

Here, $\overline{\vec{q}}_{A B}$ is the vector that connects the centroids of atoms A and $\mathrm{B}$, and $q_{A B}^{\neq}$is the distance between atoms $\mathrm{A}$ and $\mathrm{B}$ at the saddle point.

The reaction coordinate $\xi$ is defined in terms of these dividing surfaces, ${ }^{26}$

$$
\xi(\overline{\vec{q}})=\frac{s_{0}(\overline{\vec{q}})}{s_{0}(\overline{\vec{q}})-s_{1}(\overline{\vec{q}})} .
$$

Indeed, we see that $\xi \rightarrow 0$ as $s_{0} \rightarrow 0$ and $\xi \rightarrow 1$ as $s_{1} \rightarrow 0$.

To avoid computing the partition function directly, the Bennett-Chandler factorization ${ }^{30,31}$ is used,

$$
k_{R P M D}=f(T) \kappa\left(t \rightarrow \infty ; \xi^{\ddagger}\right) k_{Q T S T}\left(T ; \xi^{\ddagger}\right) .
$$

The first term is the electronic degeneracy factor, which will be discussed below. The second term represents the dynamical correction, while the third term denotes the static contribution to the rate coefficient.

In particular, $k_{Q T S T}\left(T ; \xi^{\ddagger}\right)$ is the centroid-density quantum transition-state theory (QTST) ${ }^{32,33}$ rate coefficient, calculated from the top of the free-energy barrier $\left(\xi^{\neq}\right)$along the reaction coordinate. It is determined entirely by static equilibrium properties. In practice, it is obtained from the potential of mean force (PMF) along the reaction coordinate,

$$
k_{Q T S T}\left(T ; \xi^{\ddagger}\right)=4 \pi R_{\infty}^{2}\left(\frac{1}{2 \pi \beta \mu_{R}}\right)^{1 / 2} e^{-\beta\left[W\left(\xi^{\ddagger}\right)-W(0)\right]},
$$

where $\mu_{R}$ is the reduced mass of the reactants and $W\left(\xi^{\ddagger}\right)$ $-W(0)$ is the free-energy difference between $\xi=\xi^{\ddagger}$ and $\xi=0$, which is obtained via umbrella integration along the reaction coordinate. ${ }^{34}$

The second term, $\kappa\left(t \rightarrow \infty ; \xi^{\ddagger}\right)$, namely, the transmission coefficient, provides the dynamical correction due to recrossing of the barrier. It is the ratio between the long-time limit of the flux-side correlation function and its zero-time limit,

$$
\kappa\left(t \rightarrow \infty ; \xi^{\ddagger}\right)=\frac{c_{f s}^{(n)}\left(t \rightarrow \infty ; \xi^{\ddagger}\right)}{c_{f s}^{(n)}\left(t \rightarrow 0_{+} ; \xi^{\ddagger}\right)},
$$

which accounts for recrossing at the transition state $\left(\xi=\xi^{\ddagger}\right)$. Here, the flux-side correlation function is given as ${ }^{25}$

$$
\begin{aligned}
c_{f s}^{(n)}\left(t ; \xi^{\ddagger}\right)= & \frac{1}{(2 \pi \hbar)^{n}} \int d^{n} \vec{p}_{0} \int d^{n} \vec{q}_{0} e^{-\beta_{n} H_{n}\left(\vec{p}_{0}, \vec{q}_{0}\right)} \\
& \times \delta\left(\overline{\vec{q}}_{0}-\vec{q}^{\neq}\right) \overline{\vec{v}}_{0} h\left(\overline{\vec{q}}(t)-\vec{q}^{\neq}\right),
\end{aligned}
$$

where $h$ is the Heaviside function, the subscript 0 stands for the initial position and momentum, $\overline{\vec{q}}_{0}$ denotes the initial position of the centroid of the ring polymer, and $\overline{\vec{v}}_{0}$ is the initial centroid velocity. This factor counterbalances $k_{Q T S T}\left(T ; \xi^{\ddagger}\right)$ to make sure that the RPMD rate coefficient $k_{R P M D}$ is independent of the choice of the dividing surfaces. Technically, the calculation of the transmission coefficient is to sample ring polymer trajectories with different initial momentum that start with their centroids placed at $\xi=\xi^{\ddagger}$. All RPMD calculations were performed using Suleimanov's RPMDrate program. ${ }^{26}$

The RPMD rate coefficient approaches the classical TST limit when only one bead is used, where the static and dynamical components would be equivalent to the classical TST rate coefficient and the classical transmission coefficient, respectively. When more beads are used, RPMD takes into account quantum effects such as ZPE and tunneling. The minimal number of beads needed to capture quantum effects can be estimated by the following formula: ${ }^{35}$

$$
n_{\min }=\beta \hbar \omega_{\max },
$$

where $\omega_{\max }$ is the largest vibrational frequency of the system.

Finally, the electronic degeneracy factor $f(T)$ includes the electronic and spin-orbit partition functions with the following form:

$$
f(T)=\frac{Q_{\text {elec. }}^{\mathrm{TS}}}{Q_{\text {elec. }}^{\text {Reatt }}}=\frac{6}{5+3 e^{-\beta \Delta E_{1}}+e^{-\beta \Delta E_{2}}},
$$

where $\Delta E_{1}$ and $\Delta E_{2}$ are the energies of the spin-orbit levels of $\mathrm{S}\left({ }^{3} P_{0}\right)$ and $\mathrm{S}\left({ }^{3} P_{1}\right)$ relative to that of $\mathrm{S}\left({ }^{3} P_{2}\right)$, with the experimental values 174 and $398 \mathrm{~cm}^{-1}$, respectively. The degeneracy factor $(2 J+1)$ with $J$ as the total angular momentum of the $S$ atom) takes values of $1(J=0), 3(J=1)$, and $5(J=2)$. The factor of 6 in the denominator includes the spin multiplicity of 3 and an additional factor of 2 because the $1^{3} A^{\prime}$ and $1^{3} A^{\prime \prime}$ states both contribute to the reaction and they have the same barrier height.

For comparison, variational transition-state theory calculations using the $\mathrm{CVT} / \mu \mathrm{OMT}$ method $^{36}$ are performed using POLYRATE version 2010A. ${ }^{37}$ In this method, canonical variational theory (CVT) is used to calculate rate coefficients at different temperatures. Tunneling is approximated by the microcanonical optimized multidimensional tunneling $(\mu \mathrm{OMT})$ approach,${ }^{38}$ in which the larger tunneling probability from two approximations, the small-curvature tunneling (SCT) and large-curvature ground-state tunneling (LCT), was taken as the best estimate. The step size for computing the minimum energy path (SSTEP) is set to $10^{-5}$. The same temperatures as used in RPMD calculations are selected, plus further temperatures to achieve a smooth curve.

\section{Potential energy surface}

There are two triplet states $\left(1^{3} A^{\prime}\right.$ and $\left.1^{3} A^{\prime \prime}\right)$ that facilitate the title reaction, and both have the same collinear (S-H-H) transition-state geometry and energy. ${ }^{7}$ Interestingly, the PESs of these two triplet states cross with that of the lowest singlet state, facilitating ISC. The first global PES for the $1^{3} A^{\prime \prime}$ state was developed by Maiti, Schatz, and Lendvay, ${ }^{7}$ who have also computed the spin-orbit coupling among the singlet and triplet states. The ISC dynamics have been investigated using quasi-classical trajectory surface hopping and wave packet methods. ${ }^{7,39}$ Subsequently, several 


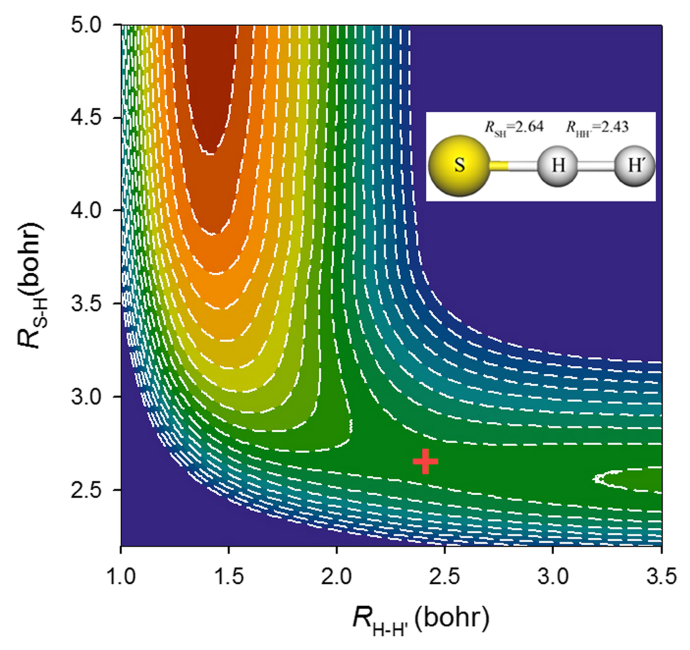

FIG. 1. Contour plot of the PIP-NN PES in the S-H and $\mathrm{H}-\mathrm{H}^{\prime}$ coordinates in collinear geometry. The transition state is indicated in the figure by a cross, and its geometry is given in the inset. The energy difference between adjacent contours is $0.1 \mathrm{eV}$.

triplet PESs have been developed from high-level $a b$ initio

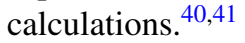

Very recently, we have carried out extensive explicitly correlated unrestricted coupled cluster theory with single, double, and perturbative triple excitations [UCCSD(T)-F12b] ${ }^{42}$ calculations on the $1^{3} A^{\prime \prime}$ PES. ${ }^{13}$ These ab initio points have been used to construct a global PES using the permutation invariant polynomial-neural network (PIP-NN) method. ${ }^{43}$ The PIP-NN method enforces the permutation symmetry rigorously and offers an extremely flexible functional form for high-fidelity representation of the $a b$ initio points. ${ }^{44}$ In particular, 3741 points were fit using a NN with 2 hidden layers of 20 neurons each and three PIPs in the input layer. These data points were randomly divided into the training (90\%), validation (5\%), and test $(5 \%)$ sets. The final fit has a root mean square fitting error of $4.3 \mathrm{meV}$. In Fig. 1, the PIP-NN PES is plotted in the collinear geometry.

\section{RESULTS AND DISCUSSION}

\section{A. Experiments}

Thirty five determinations of $k_{1}$ and nine of $k_{2}$ are summarized in Tables I and II. There is no consistent deviation of the points around the trends with respect to $\tau_{\text {res }}$ and the product $F\left[\mathrm{CS}_{2}\right]$, which indicates that $\mathrm{CS}_{2}$ is not decomposing and that there is no interference by secondary chemistry that might arise from photolysis or reaction products. The rate coefficients are plotted in the Arrhenius form in Figs. 2 and 3 and there is clear curvature. The $1 \sigma$ uncertainties listed in Tables I and II are statistical only and reflect the uncertainties in slopes of plots of $k_{p s 1}$ vs reactant concentration. To allow for the limited number of degrees of freedom in the linear plots of five $k_{p s}$ values vs reactant concentration, $1 \sigma$ values for the slopes were scaled by 1.5 before combination in quadrature with a $2 \%$ uncertainty to account for $\sigma_{\mathrm{T}} / T=2 \%{ }^{16}$ which influences the reactant concentration and then used in weighted fits to summarize the data. For reaction (1) we found that the usual modified Arrhenius expression or a sum of two Arrhenius terms does not represent the data well, and so we employ a form proposed by Zheng and Truhlar, 45

$$
k_{1}(600-1110 \mathrm{~K})=3.0 \times 10^{-9} \exp \left(-\frac{1.317 \times 10^{5}-2.703 \times 10^{7} \mathrm{~K} / T}{8.314 T / \mathrm{K}}\right) \mathrm{cm}^{3} \text { molecule }^{-1} \mathrm{~s}^{-1} .
$$

As usual, statistical confidence limits are smallest in the middle of the range and flare outwards at the extremes. Here the $95 \%$ limits are about $11 \%$ at $1100 \mathrm{~K}, 6 \%$ at $800 \mathrm{~K}$, and $33 \%$ at $600 \mathrm{~K}$. With a $5 \%$ allowance for possible systematic errors in the pressure and flow measurements, we suggest overall $95 \%$ error limits of $\pm 12 \%$ above $700 \mathrm{~K}$ and $\pm 35 \%$ below that. For reaction (2) a standard modified Arrhenius expression serves well,

$$
k_{2}(770-1110 \mathrm{~K})=2.2 \times 10^{-14}(T / 298 \mathrm{~K})^{3.55} \exp (-5420 \mathrm{~K} / T) \mathrm{cm}^{3} \text { molecule }^{-1} \mathrm{~s}^{-1} \text {. }
$$

Two- $\sigma$ statistical uncertainties in the fitted $k_{2}$ are $8 \%-13 \%$. Taking the higher values combined in quadrature with an estimated $5 \%$ allowance for systematic errors leads to $95 \%$ error limits of $\pm 14 \%$ for $k_{2}$.

The data for $\mathrm{S}+\mathrm{H}_{2}$ at $600 \mathrm{~K}$ lie somewhat above the fitted curve and well above a linear extrapolation of the hightemperature results. In principle, there could be several causes including operation of a second reaction channel, tunneling, and low frequency modes in the transition state—factors which are addressed in Sec. III B-and interference by secondary chemistry when the prime reaction becomes very slow as here, ca. $10^{-16} \mathrm{~cm}^{3}$ molecule $\mathrm{e}^{-1} \mathrm{~s}^{-1}$. Evidence against such interference is that lowering $[\mathrm{S}]_{0}$ by a factor of $5 \mathrm{did}$ not reduce the observed rate coefficient. Further, the fluorescence decays were exponential within the expected scatter of the photon counts. Nevertheless, we apply another way to assess the experiments, by modeling some further chemistry of the reaction products, i.e.,

$$
\begin{aligned}
\mathrm{S}+\mathrm{SH} & \rightarrow \mathrm{S}_{2}+\mathrm{H}, \\
\mathrm{H}+\mathrm{SH} & \rightarrow \mathrm{H}_{2}+\mathrm{S}, \\
\mathrm{SH}+\mathrm{SH} & \rightarrow \mathrm{H}_{2} \mathrm{~S}+\mathrm{S} .
\end{aligned}
$$

Proposed values of the rate coefficients are $4 \times 10^{-11}$, $3 \times 10^{-11}$, and $1.5 \times 10^{-11} \mathrm{~cm}^{3}$ molecule ${ }^{-1} \mathrm{~s}^{-1}$, respectively. ${ }^{46}$ If every $\mathrm{SH}$ produced in reaction (1) quickly consumed a second $\mathrm{S}$ atom via reaction (18), then the observed rate coefficient for S-atom loss would be double the true value of $k_{1}$. Using 
TABLE I. Summary of measurements on $\mathrm{S}\left({ }^{3} \mathrm{P}\right)+\mathrm{H}_{2}$.

\begin{tabular}{|c|c|c|c|c|c|c|c|c|}
\hline$T / \mathrm{K}$ & $\tau_{\text {res }} / \mathrm{s}$ & $F / \mathrm{mJ} \mathrm{cm}^{-2}$ & $P /$ mbar & $\begin{array}{c}{\left[\mathrm{H}_{2}\right]_{\max } / 10^{17}} \\
\text { molecule } \\
\mathrm{cm}^{-3}\end{array}$ & $\begin{array}{c}{\left[\mathrm{CS}_{2}\right] / 10^{13}} \\
\text { molecule } \\
\mathrm{cm}^{-3}\end{array}$ & $\begin{array}{c}{[\mathrm{S}]_{0} / 10^{12}} \\
\text { molecule } \\
\mathrm{cm}^{-3}\end{array}$ & $\begin{array}{c}k_{1} / 10^{-15} \\
\text { molecule }^{-1} \\
\mathrm{~cm}^{3} \mathrm{~s}^{-1}\end{array}$ & $\begin{array}{c}\sigma_{k_{1}} / 10^{-15} \\
\text { molecule } \\
\mathrm{cm}^{3} \mathrm{~s}^{-1}\end{array}$ \\
\hline 601 & 1.48 & 0.08 & 68 & 2.05 & 6.64 & 0.67 & 0.11 & 0.01 \\
\hline 601 & 2.09 & 0.08 & 268 & 2.95 & 9.42 & 0.95 & 0.12 & 0.01 \\
\hline 601 & 1.49 & 0.03 & 69 & 2.06 & 6.99 & 0.19 & 0.12 & 0.01 \\
\hline 641 & 1.86 & 1.12 & 133 & 3.05 & 4.47 & 5.23 & 0.16 & 0.02 \\
\hline 641 & 1.86 & 0.58 & 133 & 3.05 & 4.47 & 3.28 & 0.16 & 0.01 \\
\hline 650 & 1.90 & 0.11 & 137 & 2.66 & 6.61 & 0.93 & 0.18 & 0.01 \\
\hline 650 & 1.16 & 0.11 & 135 & 1.64 & 4.03 & 0.57 & 0.19 & 0.03 \\
\hline 650 & 2.28 & 0.11 & 268 & 3.22 & 7.99 & 1.13 & 0.21 & 0.01 \\
\hline 716 & 1.31 & 0.66 & 267 & 1.84 & 4.25 & 3.56 & 0.38 & 0.01 \\
\hline 716 & 1.31 & 0.31 & 267 & 1.84 & 4.25 & 1.63 & 0.39 & 0.03 \\
\hline 720 & 1.03 & 0.52 & 133 & 1.27 & 2.53 & 1.68 & 0.41 & 0.02 \\
\hline 720 & 1.03 & 0.33 & 133 & 1.27 & 2.53 & 1.06 & 0.42 & 0.02 \\
\hline 721 & 2.55 & 0.93 & 267 & 3.53 & 4.27 & 4.98 & 0.44 & 0.01 \\
\hline 721 & 2.55 & 0.44 & 267 & 3.53 & 4.27 & 2.40 & 0.43 & 0.01 \\
\hline 721 & 2.55 & 0.27 & 267 & 3.53 & 4.27 & 1.44 & 0.42 & 0.01 \\
\hline 750 & 0.80 & 0.10 & 67 & 1.14 & 4.93 & 0.58 & 0.79 & 0.05 \\
\hline 750 & 1.61 & 0.10 & 134 & 2.25 & 7.25 & 0.85 & 0.73 & 0.02 \\
\hline 750 & 1.00 & 0.10 & 134 & 1.39 & 6.06 & 0.71 & 0.70 & 0.03 \\
\hline 823 & 1.46 & 0.81 & 133 & 2.41 & 3.53 & 3.62 & 1.61 & 0.09 \\
\hline 823 & 1.46 & 0.52 & 133 & 2.41 & 3.53 & 2.34 & 1.68 & 0.08 \\
\hline 824 & 1.52 & 0.56 & 267 & 2.53 & 3.67 & 2.58 & 1.43 & 0.02 \\
\hline 824 & 1.52 & 0.35 & 267 & 2.53 & 3.67 & 1.57 & 1.45 & 0.01 \\
\hline 920 & 1.35 & 0.68 & 267 & 0.96 & 2.60 & 2.23 & 5.01 & 0.11 \\
\hline 920 & 1.35 & 0.35 & 267 & 0.96 & 2.60 & 1.14 & 5.07 & 0.07 \\
\hline 920 & 1.36 & 0.68 & 267 & 0.96 & 4.99 & 4.72 & 5.04 & 0.02 \\
\hline 922 & 0.80 & 0.73 & 133 & 0.85 & 2.95 & 2.73 & 6.10 & 0.07 \\
\hline 923 & 1.57 & 0.47 & 133 & 1.38 & 5.66 & 3.36 & 5.79 & 0.40 \\
\hline 923 & 1.35 & 0.54 & 67 & 0.70 & 2.42 & 1.65 & 6.03 & 0.15 \\
\hline 1028 & 1.36 & 0.68 & 267 & 1.52 & 3.47 & 2.96 & 10.46 & 0.19 \\
\hline 1028 & 0.59 & 0.42 & 267 & 1.52 & 3.47 & 1.82 & 11.47 & 0.20 \\
\hline 1028 & 0.61 & 0.95 & 133 & 1.02 & 3.37 & 4.07 & 13.46 & 0.17 \\
\hline 1028 & 0.61 & 0.58 & 133 & 1.02 & 3.37 & 2.49 & 14.50 & 0.14 \\
\hline 1031 & 0.90 & 0.85 & 67 & 0.83 & 3.25 & 3.48 & 15.95 & 1.31 \\
\hline 1109 & 0.90 & 0.58 & 267 & 0.64 & 3.42 & 2.49 & 22.10 & 1.15 \\
\hline 1109 & 0.90 & 0.37 & 267 & 0.64 & 3.42 & 1.61 & 25.47 & 0.92 \\
\hline
\end{tabular}

the conditions of Table I and modeling reactions (1) and (18)(20), at $600 \mathrm{~K}$ we find that effective rate coefficients for the first 1/e lifetime of [S] would increase by less than $10 \%$ over the range of $[\mathrm{S}]_{0}$ used.
Our rate coefficients compare well with prior data obtained in shock tube experiments. ${ }^{4,5,15}$ Evaluation of the various rate coefficient expressions at intervals of $1000 \mathrm{~K} / T=0.01$ yields combined data sets, which can be summarized by

TABLE II. Summary of measurements on $\mathrm{S}\left({ }^{3} \mathrm{P}\right)+\mathrm{D}_{2}$.

\begin{tabular}{|c|c|c|c|c|c|c|c|c|}
\hline$T / \mathrm{K}$ & $\tau_{\text {res }} / \mathrm{s}$ & $F / \mathrm{mJ} \mathrm{cm}^{-2}$ & $P / \mathrm{mbar}$ & $\begin{array}{c}{\left[\mathrm{D}_{2}\right]_{\max } / 10^{17}} \\
\text { molecule } \\
\mathrm{cm}^{-3}\end{array}$ & $\begin{array}{c}{\left[\mathrm{CS}_{2}\right] / 10^{13}} \\
\text { molecule } \\
\mathrm{cm}^{-3}\end{array}$ & $\begin{array}{c}{[\mathrm{S}]_{0} / 10^{11}} \\
\text { molecule } \\
\mathrm{cm}^{-3}\end{array}$ & $\begin{array}{c}k_{2} / 10^{-15} \\
\text { molecule } \\
\mathrm{cm}^{3} \mathrm{~s}^{-1}\end{array}$ & $\begin{array}{c}\sigma_{k_{2}} / 10^{-15} \\
\text { molecule } \\
\mathrm{cm}^{3} \mathrm{~s}^{-1}\end{array}$ \\
\hline 772 & 0.80 & 0.061 & 68 & 0.91 & 4.40 & 3.38 & 0.57 & 0.050 \\
\hline 772 & 1.57 & 0.061 & 134 & 2.38 & 6.39 & 4.90 & 0.61 & 0.029 \\
\hline 772 & 1.62 & 0.061 & 268 & 2.49 & 6.61 & 5.07 & 0.51 & 0.035 \\
\hline 821 & 0.75 & 0.076 & 67 & 1.00 & 3.02 & 2.90 & 1.03 & 0.068 \\
\hline 821 & 1.51 & 0.076 & 135 & 2.01 & 6.08 & 5.83 & 1.15 & 0.080 \\
\hline 921 & 0.66 & 0.076 & 68 & 0.76 & 2.69 & 2.58 & 3.10 & 0.247 \\
\hline 921 & 1.31 & 0.076 & 134 & 1.51 & 5.39 & 5.17 & 3.30 & 0.125 \\
\hline 1018 & 1.56 & 0.061 & 133 & 0.90 & 4.81 & 3.69 & 9.33 & 0.710 \\
\hline 1106 & 1.16 & 0.113 & 269 & 1.13 & 2.67 & 3.82 & 16.71 & 0.761 \\
\hline
\end{tabular}




$$
k_{1}(600-3140 \mathrm{~K})=2.0 \times 10^{-9} \exp \left(-\frac{1.24 \times 10^{5}-2.34 \times 10^{7} \mathrm{~K} / T}{8.314 T / \mathrm{K}}\right) \mathrm{cm}^{3} \text { molecule }^{-1} \mathrm{~s}^{-1}
$$

and

$$
k_{2}(770-1680 \mathrm{~K})=8.1 \times 10^{-10} \exp \left(-\frac{1.17 \times 10^{5}-2.06 \times 10^{7} \mathrm{~K} / T}{8.314 T / \mathrm{K}}\right) \mathrm{cm}^{3} \text { molecule }^{-1} \mathrm{~s}^{-1} .
$$

\section{B. Theoretical analysis}

The parameters used in the RPMD calculations are listed in Table III.

Figure 4 shows the comparison of the RPMD rate coefficients of the $\mathrm{S}+\mathrm{H}_{2}$ reaction with experimental and other theoretical results. The calculated data are all based on the newly developed PES described in Sec. II C. The classical TST rate coefficients (green open squares and solid line) were obtained by RPMD with only one bead. As expected, the corresponding rate coefficients form a straight line in the Arrhenius plot, suggesting the absence of tunneling. These classical results agree with the converged RPMD results (with optimum numbers of beads, blue filled circles and solid line) well at high temperatures, where tunneling is not important. However, the single- and multi-bead RPMD results deviate more with decreasing temperature, suggesting the ever-growing importance of tunneling at lower temperatures. The CVT/MOMT results plotted in the same figure (orange filled diamonds and dashed line) are very close to the converged RPMD ones, although slightly higher than their RPMD counterparts.

While the agreement with the measured rate coefficients near $1000 \mathrm{~K}$ is quite good, the RPMD and CVT/ $\mu \mathrm{OMT}$ results underestimate the experiment significantly at lower temperatures. As discussed above, this deviation is not due to tunneling, which has been taken into consideration already. The culprit is apparently the fact that the RPMD and CVT/ $\mu$ OMT calculations were all done with the assumption that the reaction takes place on triplet state PESs. Yet, previous theoretical studies have indicated that the title reaction is strongly influenced by ISC between the triplet and singlet states, thanks to the large spin-orbit coupling of the heavy $\mathrm{S}$ atom. ${ }^{7,39}$ The singlet PES nominally facilitating the $\mathrm{S}\left({ }^{1} \mathrm{D}\right)+\mathrm{H}_{2} \rightarrow \mathrm{SH}+\mathrm{H}$ reaction,

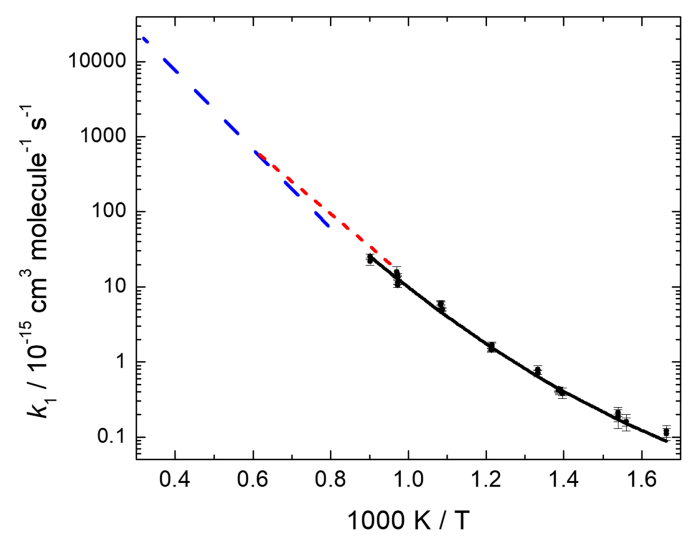

FIG. 2. Arrhenius plot for the $\mathrm{S}+\mathrm{H}_{2}$ reaction. Solid line and points with $2 \sigma$ error bars-present work; short dashes-Shiina et al. $;^{4}$ long dashes-Woiki and Roth. ${ }^{5}$ which shares the same product channel, is barrierless. As a result, the $\mathrm{S}\left({ }^{3} \mathrm{P}\right)+\mathrm{H}_{2} \rightarrow \mathrm{SH}+\mathrm{H}$ reaction, which has a significant barrier, could gain substantial reactivity through ISC via triplet-singlet surface crossings. This should be particularly important for low collision energies/low temperatures for the title reaction as the indirect ISC presents a lower effective potential barrier. ${ }^{7}$ At high temperatures, on the other hand, the more effective pathway is the direct one on the triplet PESs, which explains the good agreement with the high-temperature experimental data.

Figure 5 shows rate coefficients of the $\mathrm{S}+\mathrm{D}_{2}$ reaction. Again, the theoretical results are in good agreement between themselves and with the experiment at high temperatures (e.g., $\sim 1000 \mathrm{~K}$ ) but underestimate the measured rate coefficients at lower temperatures. In this reaction, the tunneling contribution is expected to be less than reaction (1), but the substantial theory-experiment differences persist at low temperatures. Again, the theory-experiment deviation is attributed to the neglect of ISC in our calculations.

It would be interesting if the non-adiabatic ISC can be simulated in the same RPMD framework to quantify its impact on the rate coefficients. Despite some attempts, ${ }^{47-49}$ however, a reliable RPMD rate theory for treating non-adiabatic reactions has not been established. Hence, a complete treatment of the kinetics of the title reactions will have to wait for future method developments.

The kinetic isotope effects (KIEs) are displayed in Fig. 6. For $T$ in the approximate range 700-1100 K, the KIE is based on our own measurements, while for $1200 \mathrm{~K}$ and up, we use the ratio of the combined datasets via Eqs. (21) and (22). An alternative derivation of the KIE at high temperatures is via the shock tube of the Matsui group alone. ${ }^{15}$ This latter treatment increases the apparent KIE by a factor close to 1.5 at $\sim 1200 \mathrm{~K}$ because $k_{1}$ from the work of Shiina et al. ${ }^{4}$ is

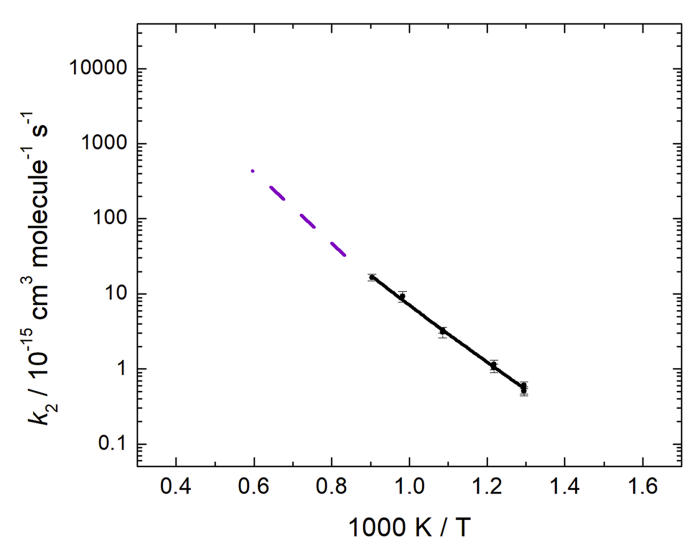

FIG. 3. Arrhenius plot for the $\mathrm{S}+\mathrm{D}_{2}$ reaction. Solid line and points with $2 \sigma$ error bars-present work; dashes-Tsuchiya et al. $^{15}$ 
TABLE III. Parameters for the RPMD rate coefficient calculations on the $\mathrm{S}+\mathrm{H}_{2}$ reaction.

\begin{tabular}{|c|c|c|}
\hline Parameter & Values & Note \\
\hline & Command line parameters & \\
\hline$T / \mathrm{K}$ & $500,600,821,921,1100,3140$ & Temperature \\
\hline \multirow[t]{2}{*}{$n$} & $64,64,16,8,8,1$ & Number of beads \\
\hline & Dividing surface parameters & \\
\hline$R_{\infty} / a_{0}$ & 23 & Dividing surface $s_{1}$ parameter \\
\hline$N_{\text {bonds }}$ & 1 & Number of forming and breaking bonds \\
\hline \multirow[t]{2}{*}{$N_{\text {channel }}$} & 2 & Number of equivalent product channels \\
\hline & Thermostat & \\
\hline \multirow[t]{2}{*}{ Thermostat } & Andersen & Thermostat option \\
\hline & Biased sampling parameters & \\
\hline$N_{\text {windows }}$ & 110 & Number of windows \\
\hline$\xi_{i}$ & -0.04 & Center of the first window \\
\hline $\mathrm{d} \xi$ & 0.01 & Window spacing step \\
\hline$\xi_{N}$ & 1.05 & Center of the last window \\
\hline $\mathrm{d} t / \mathrm{ps}$ & $10^{-4}$ & Time step \\
\hline$k_{i}$ & $2.727(T / \mathrm{K}) \mathrm{eV}$ & Umbrella force constant \\
\hline$N_{\text {trajectory }}$ & 100 & Number of trajectories \\
\hline$t_{\text {equilibration }} / \mathrm{ps}$ & 20 & Equilibration period \\
\hline$t_{\text {sampling }} / \mathrm{ps}$ & 200 & Sampling period in each trajectory \\
\hline \multirow[t]{2}{*}{$N_{i}$} & $2 \times 10^{8}$ & Total number of sampling points \\
\hline & Potential of mean force calculation & \\
\hline$\xi_{0}$ & -0.02 & Start of umbrella integration \\
\hline$\xi_{f}$ & 1.04 & End of umbrella integration \\
\hline \multirow[t]{2}{*}{$N_{\text {bins }}$} & 5000 & Number of bins \\
\hline & Recrossing factor calculation & \\
\hline $\mathrm{d} t / \mathrm{ps}$ & 0.0001 & Time step \\
\hline$t_{\text {equilibration }} / \mathrm{ps}$ & 20 & Equilibration period in the constrained (parent) trajectory \\
\hline$N_{\text {totalchild }}$ & 200000 & Total number of unconstrained (child) trajectories \\
\hline$t_{\text {childsampling }} / \mathrm{ps}$ & 2 & Sampling increment along the parent trajectory \\
\hline$N_{\text {child }}$ & 200 & Number of child trajectories per one initially constrained configuration \\
\hline$t_{\text {child }} / \mathrm{ps}$ & 2 & Length of child trajectories \\
\hline
\end{tabular}

larger than from the work of Woiki and Roth. ${ }^{5}$ While the two approaches converge near $1600 \mathrm{~K}$, the differences at lower temperatures suggest that the experimental data still contain significant uncertainty. On the theory side, results from converged RPMD and CVT/MOMT also differ in the temperature range used in the calculation, which underscores the

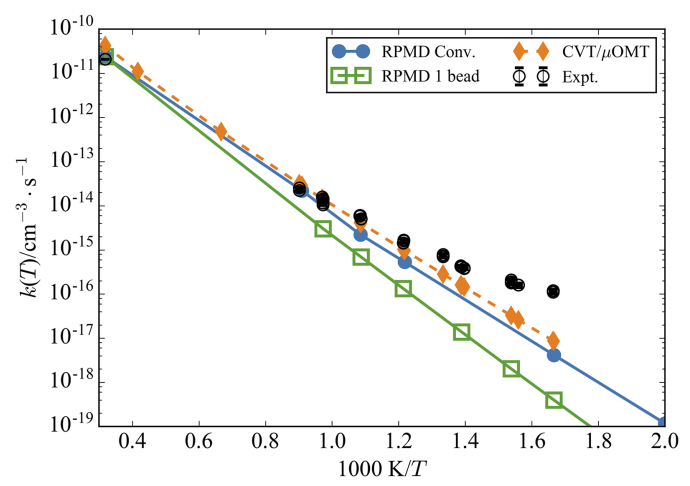

FIG. 4. Comparison of rate coefficients obtained by RPMD (Conv. denotes the converged number of beads, and 1 bead denotes that a single bead is used), $\mathrm{CVT} / \mu \mathrm{OMT}$, and experiments for the $\mathrm{S}+\mathrm{H}_{2}$ reaction. differences in treating the multidimensional tunneling. Indeed, our recent comparison between KIEs computed using RPMD and CVT for another prototypical reaction has shown large differences ${ }^{50}$ Since high temperature reactivity is essentially due to the direct channel on the triplet state PESs, the agreement

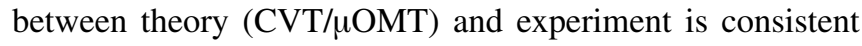

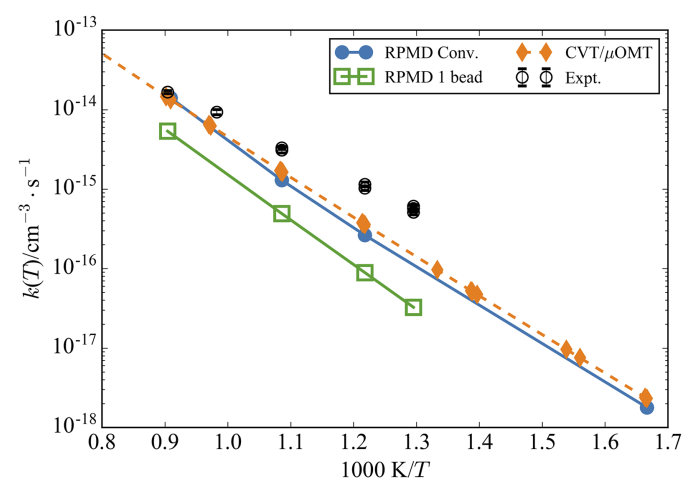

FIG. 5. Comparison among RPMD (Conv. denotes the converged number

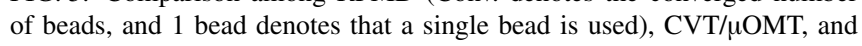
experimental rate coefficients for the $\mathrm{S}+\mathrm{D}_{2}$ reaction. 


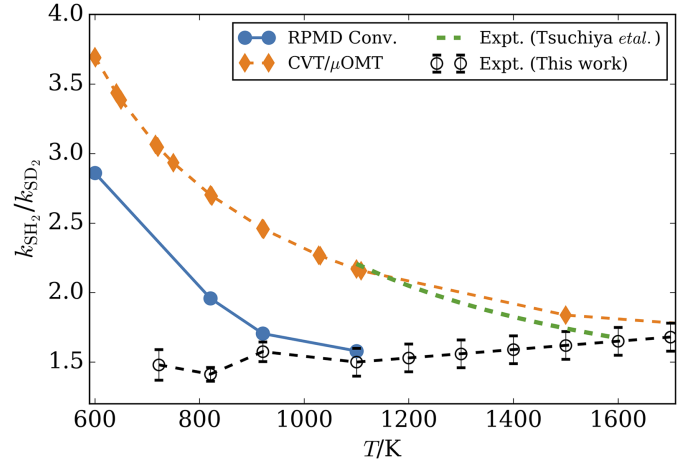

FIG. 6. Comparison of KIEs $\left(k_{\mathrm{SH}_{2}} / k_{\mathrm{SD}_{2}}\right)$ from calculations using RPMD, $\mathrm{CVT} / \mu \mathrm{OMT}$, and measurements.

with expectation. At lower temperatures, the neglect of ISC renders the theory-experiment comparison less meaningful, but the large theory-experiment differences at low temperatures might indicate an isotope dependence on the ISC. The comparison will have to wait for more sophisticated theoretical treatments that include the ISC channel.

\section{SUMMARY AND CONCLUSIONS}

Rate coefficients for $\mathrm{S}\left({ }^{3} \mathrm{P}\right)$ atoms reacting with $\mathrm{H}_{2}$ and $\mathrm{D}_{2}$ have been measured over $600-1100 \mathrm{~K}$. There is good agreement with higher temperature shock tube data in the literature. At high temperatures, the rate coefficients and KIE values from RPMD based on an abstraction pathway along the triplet PES show good coincidence with the experimental ones. At lower temperatures, however, the overall rate coefficients are heavily underestimated by this theoretical treatment. The major cause of this deviation is likely the non-adiabatic feature of the title reaction via ISC, and a corresponding version of RPMD which includes surface-hopping to the singlet PES is needed to deal with this kind of reaction.

\section{ACKNOWLEDGMENTS}

P.M. thanks the R. A. Welch Foundation (Grant No. B1174 ) and the National Science Foundation (Grant No. CBET0756144) for support and Nicole M. Wallace for assistance with the experiments. Y.L. thanks the National Natural Science Foundation of China (Grant Nos. 201503130 and 11674212). Y.L. is also supported by the Young Eastern Scholar Program of the Shanghai Municipal Education Commission (Grant No. QD2016021) and Shanghai Key Laboratory of High Temperature Superconductors (Grant No. 14DZ2260700). Y.L. also acknowledges Shanghai Supercomputer Center for CPU hours. H.G. acknowledges partial support from the Department of Energy (Grant No. DE-SC0015997).

\footnotetext{
${ }^{1}$ W. Nimmo, E. Hampartsoumian, and B. M. Gibbs, Fuel 80, 887-897 (2001). ${ }^{2}$ P. Glarborg, Proc. Combust. Inst. 31, 77-98 (2007).

${ }^{3}$ M. Binoist, B. Labégorre, F. Monnet, P. D. Clark, N. I. Dowling, M. Huang, D. Archambault, E. Plasari, and P.-M. Marquaire, Ind. Eng. Chem. Res. 42, 3943-3951 (2003).

${ }^{4}$ H. Shiina, M. Oya, K. Yamashita, A. Miyoshi, and H. Matsui, J. Phys. Chem. 100, 2136-2140 (1996).

${ }^{5}$ D. Woiki and P. Roth, Int. J. Chem. Kinet. 27, 547-553 (1995).
}

${ }^{6}$ H. Shiina, A. Miyoshi, and H. Matsui, J. Phys. Chem. 102, 3556-3559 (1998).

${ }^{7}$ B. Maiti, G. C. Schatz, and G. Lendvay, J. Phys. Chem. A 108, 8772-8781 (2004).

${ }^{8}$ T. Higashihara, K. Saito, and H. Yamamura, Bull. Chem. Soc. Jpn. 49, 965-968 (1976).

${ }^{9}$ C. T. Bowman and L. G. Dodge, Proc. Combust. Inst. 16, 971-982 (1976).

${ }^{10}$ P. Roth, R. Löhr, and U. Barner, Combust. Flame 45, 273-285 (1982).

${ }^{11}$ D. Woiki and P. Roth, J. Phys. Chem. 98, 12958-12963 (1994).

${ }^{12}$ H. A. Olschewski, J. Troe, and H. G. Wagner, J. Phys. Chem. 98, 12964 12967 (1994).

${ }^{13}$ B. Kolb, P. Marshall, B. Zhao, B. Jiang, and H. Guo, J. Phys. Chem. A 121, 2552-2557 (2017)

${ }^{14}$ Y. V. Suleimanov, F. J. Aoiz, and H. Guo, J. Phys. Chem. A 120, 8488-8502 (2016).

${ }^{15}$ K. Tsuchiya, K. Yamashita, A. Miyoshi, and H. Matsui, J. Phys. Chem. 100, 17202-17206 (1996).

${ }^{16}$ L. Ding and P. Marshall, J. Chem. Soc., Faraday Trans. 89, 419-423 (1993).

${ }^{17}$ Y. Shi and P. Marshall, J. Phys. Chem. 95, 1654-1658 (1991).

${ }^{18}$ A. Goumri, D. D. Shao, and P. Marshall, J. Chem. Phys. 121, 9999-10005 (2004).

${ }^{19}$ G. Black and L. E. Jusinski, J. Chem. Phys. 82, 789-793 (1985).

${ }^{20}$ Y. Gao and P. Marshall, J. Chem. Phys. 135, 144306 (2011).

${ }^{21}$ S. Habershon, D. E. Manolopoulos, T. E. Markland, and T. F. Miller III, Annu. Rev. Phys. Chem. 64, 387-413 (2013).

${ }^{22}$ I. R. Craig and D. E. Manolopoulos, J. Chem. Phys. 122, 084106 (2005).

${ }^{23}$ I. R. Craig and D. E. Manolopoulos, J. Chem. Phys. 123, 034102 (2005).

${ }^{24}$ R. Collepardo-Guevara, Y. V. Suleimanov, and D. E. Manolopoulos, J. Chem. Phys. 130, 174713 (2009).

${ }^{25}$ Y. V. Suleimanov, R. Collepardo-Guevara, and D. E. Manolopoulos, J. Chem. Phys. 134, 044131 (2011).

${ }^{26}$ Y. V. Suleimanov, J. W. Allen, and W. H. Green, Comput. Phys. Commun. 184, 833-840 (2013).

${ }^{27}$ I. R. Craig and D. E. Manolopoulos, J. Chem. Phys. 121, 3368-3373 (2004).

${ }^{28}$ T. J. H. Hele and S. C. Althorpe, J. Chem. Phys. 139, 084115 (2013).

${ }^{29}$ T. J. H. Hele and S. C. Althorpe, J. Chem. Phys. 138, 084108 (2013).

${ }^{30} \mathrm{C}$. H. Bennett, in Algorithms for Chemical Computations, ACS Symposium Series, edited by R. E. Christofferson (ACS, 1977), Vol. 46.

${ }^{31}$ D. Chandler, J. Chem. Phys. 68, 2959-2970 (1978).

${ }^{32}$ M. J. Gillan, J. Phys. C 20, 3621-3641 (1987).

${ }^{33}$ G. A. Voth, D. Chandler, and W. H. Miller, J. Chem. Phys. 91, 7749-7760 (1989)

${ }^{34}$ J. Kästner and W. Thiel, J. Chem. Phys. 123, 144104 (2005).

${ }^{35}$ T. E. Markland and D. E. Manolopoulos, J. Chem. Phys. 129, 024105 (2008).

${ }^{36}$ D. G. Truhlar and B. C. Garrett, Annu. Rev. Phys. Chem. 35(1), 159-189 (1984)

${ }^{37}$ J. Zheng, J. L. Bao, R. Meana-Pañeda, S. Zhang, B. J. Lynch, J. C. Corchado, Y.-Y. Chuang, P. L. Fast, W.-P. Hu, Y.-P. Liu, G. C. Lynch, K. A. Nguyen, C. F. Jackels, A. F. Ramos, B. A. Ellingson, V. S. Melissas, J. Villà, I. Rossi, E. L. Coitiño, J. Pu, T. V. Albu, A. Ratkiewicz, R. Steckler, B. C. Garrett, A. D. Isaacson, and D. G. Truhlar, POLYRATE-version 2016, University of Minnesota, Minneapolis, 2016.

${ }^{38}$ Y.-P. Liu, D.-H. Lu, A. Gonzalez-Lafont, D. G. Truhlar, and B. C. Garrett, J. Am. Chem. Soc. 115, 7806-7817 (1993).

${ }^{39}$ T.-S. Chu, K.-L. Han, and G. C. Schatz, J. Phys. Chem. A 111, 8286-8290 (2007).

${ }^{40}$ S.-J. Lv, P.-Y. Zhang, K.-L. Han, and G.-Z. He, J. Chem. Phys. 136, 094308 (2012).

${ }^{41}$ S.-J. Lv, P.-Y. Zhang, and G.-Z. He, Chin. J. Chem. Phys. 25, 291-296 (2012).

${ }^{42}$ G. Knizia, T. B. Adler, and H.-J. Werner, J. Chem. Phys. 130, 054104 (2009).

${ }^{43}$ B. Jiang and H. Guo, J. Chem. Phys. 139, 054112 (2013).

${ }^{44}$ B. Jiang, J. Li, and H. Guo, Int. Rev. Phys. Chem. 35, 479-506 (2016).

${ }^{45}$ J. Zheng and D. G. Truhlar, Phys. Chem. Chem. Phys. 12, 7782-7793 (2010).

${ }^{46}$ K. Schofield, J. Phys. Chem. Ref. Data 2, 25-84 (1973).

${ }^{47}$ P. Shushkov, R. Li, and J. C. Tully, J. Chem. Phys. 137, 22 A549 (2012).

${ }^{48}$ J. O. Richardson and M. Thoss, J. Chem. Phys. 139, 031102 (2013).

${ }^{49}$ A. R. Menzeleev, F. Bell, and T. F. Miller III, J. Chem. Phys. 140, 064103 (2014)

${ }^{50}$ J. Zuo, C. Xie, H. Guo, and D. Xie, J. Phys. Chem. Lett. 8, 3392-3397 (2017). 